\title{
Management of Postpartum Hemorrhage: Fibrinogen vs. Prothrombin Complex concentrate
}

\author{
Ata Mahmoodpoor ${ }^{1^{*}}$, Sarvin Sanaie ${ }^{2}$
}

$\mathrm{P}$ ostpartum hemorrhage $(\mathrm{PPH})$ is the leading cause of maternal mortality. All women with a pregnancy beyond 20 weeks of gestational age are at risk of PPH and its sequelae. Although maternal mortality rates have greatly declined in the developed world, $\mathrm{PPH}$ remains a leading cause of maternal mortality. Incidence of PPH has recently increased in some countries which can be related to the increased use of oxytocin for labor augmentation and subsequent uterine atony (1). As members of the multidisciplinary team, critical care physicians should improve their expertise in fluid management, transfusion therapy, and intensive care management to prevent and treat the catastrophic events of PPH. There are many techniques for managing $\mathrm{PPH}$ (many guidelines and institution-specific protocols), some of which may reduce the incidence of maternal morbidity and mortality (2). Until now, no single trial has proven that a specific transfusion strategy or the use of any prohemostatic agent can improve maternal outcomes after $\mathrm{PPH}$ (3). Considering changes in maternal coagulation status during major $\mathrm{PPH}$, fibrinogen can be an important therapeutic target. For treating hypofibrinogemia, we have three options: FFP (fresh frozen plasma), cryo and fibrinogen. The first two need larger volume for appropriate amount of fibrinogen replacement and also need blood type matching and thawing which may lead to significant time delay during management of major blood loss. Fibrinogen, as a glycoprotein, has some roles in hemostasis such as increasing platelet aggregation and activation and secondary hemostasis defined as starting fibrin polymerization after undergoing cleavage $(4,5)$. Many studies have shown that low fibrinogen level $(<2$ $\mathrm{g} / \mathrm{L}$ ) is an independent predictor for PPH mortality (69). Results of two studies in which thromboelastography was used for management of $\mathrm{PPH}$ showed that functional fibrinogen deficiency could worsen the outcome of major $\mathrm{PPH}(9,10)$. If fibrinogen therapy is guided by results of ROTEM (Rotational thromboelastography), it will lead to reduced requirements for blood components and lower risk of circulatory overload (11). European society of anesthesiologists recommended an initial fibrinogen concentrate dose of $20-25 \mathrm{~mL} / \mathrm{kg}$ in plasma fibrinogen concentration of less than $1.5-2 \mathrm{~g} / \mathrm{L}$ or thromboelastography signs of functional fibrinogen deficit (12). Results of a recent multicenter trial recommended fibrinogen concentrate administration when fibrinogen levels are below 3.0 to $4.0 \mathrm{~g} / \mathrm{L}$ in the presence of active bleeding (13); however, higher trigger levels $(<4.0 \mathrm{~g} / \mathrm{L})$ were suggested in another study (14). It is reported that fibrinogen levels $\leq 2 \mathrm{~g} / \mathrm{L}$ have a positive predictive value of $100 \%$ and levels $>4 \mathrm{~g} / \mathrm{L}$ have a negative predictive value of $79 \%$ for the development of severe PPH. A large recent multicenter trial failed to show the beneficial effect of preemptive administration of $2 \mathrm{~g}$ fibrinogen concentrate on PPH in normofibrinogenemia patients (15).

There are two types of prothrombin concentrate complex (PCC): a three-factor PCC and a four-factor PCC including factor VII. PCC is recommended for rapid reversal of warfarin therapy or vitamin $\mathrm{K}$ deficiency in patients exhibiting major bleeding manifestations and rapid reversal of warfarin therapy or vitamin $\mathrm{K}$ deficiency in patients requiring urgent ( $<6$ hours) surgical procedures. As there are so many types of PCC with different dosage of composed factors, reaching an evidence-based approach is so difficult. There is insufficient published evidence available to allow a recommendation for the use or dosing of these products in PPH. Caution should be exercised if they are used in pregnancy, particularly in the peripartum/ early postpartum period because of a heightened tendency to thrombosis (16).

Determining the true effect of fibrinogen concentrate or PCC is influenced by heterogeneity in hemorrhage etiology, the use of high volumes of colloidal solutions and the transfusion of other plasma derived concentrates, prothrombotics and antifibrinolytics within the similar time period (17). Based on predisposing factors and $\mathrm{PPH}$ etiology, more comparative effectiveness research is needed to assess the potential benefits of fibrinogen concentrates for $\mathrm{PPH}$ compared to more traditional approaches to fibrinogen replacement. Prompt and effective management, multidisciplinary involvement, more advanced coagulation monitoring and bedside assessment of the maternal coagulation profile can help physicians to provide more personalized goal-directed 
therapy for patients with PPH. The timely and rational use of coagulation factor concentrates may be more efficacious and safer than ratio-driven use of transfusion packages of allogeneic blood. Finally, fibrinogen appears to be a promising therapeutic option for PPH but there is no strong evidence for its routine use in the literature; on the other hand, lack of thromboembolic complications after fibrinogen is ideal. A large multicenter randomized double blinded trial is being performed with the aim of evaluating the effect of early administration of 3 $\mathrm{g}$ fibrinogen on PPH-induced coagulopathy in 412 patients (FIDEL trial) whose result can help for the better management of PPH in the future (18). The evidence for PCC in PPH is limited to case reports and the incidence of thromboembolic complications seems to be increased following its usage.

Therefore, despite the lack of conclusive evidence for optimal hemostatic resuscitation in $\mathrm{PPH}$, viscoelastic hemostatic assays and appropriate fibrinogen usage may be potentially pivotal in the treatment of $\mathrm{PPH}$.

\section{Ethical Issues}

Not applicable.

\section{Conflict of Interests}

The authors declare no conflict of interests.

\section{Financial Support}

None.

\section{References}

1. Belghiti J, Kayem G, Dupont C, Rudigoz RC, BouvierColle MH, Deneux-Tharaux C. Oxytocin during labour and risk of severe postpartum haemorrhage: a populationbased, cohort-nested case-control study. BMJ Open. 2011;1(2):e000514. doi: 10.1136/bmjopen-2011-000514.

2. Shields LE, Smalarz K, Reffigee L, Mugg S, Burdumy TJ, Propst M. Comprehensive maternal hemorrhage protocols improve patient safety and reduce utilization of blood products. Am J Obstet Gynecol. 2011;205(4):368.e1-8. doi: 10.1016/j.ajog.2011.06.084.

3. Bonnet MP, Benhamou D. Management of postpartum haemorrhage. F1000Res. 2016;5. doi: 10.12688/ f1000research.7836.1.

4. Weisel JW, Nagaswami C, Vilaire G, Bennett JS. Examination of the platelet membrane glycoprotein IIb-IIIa complex and its interaction with fibrinogen and other ligands by electron microscopy. J Biol Chem. 1992;267(23):16637-43.

5. Mosesson MW. Fibrinogen and fibrin structure and functions. J Thromb Haemost. 2005;3(8):1894-904. doi: 10.1111/j.1538-7836.2005.01365.x.

6. Charbit B, Mandelbrot L, Samain E, et al. The decrease of fibrinogen is an early predictor of the severity of postpartum hemorrhage. J Thromb Haemost. 2007;5(2):266-73. doi: 10.1111/j.1538-7836.2007.02297.x.

7. Cortet M, Deneux-Tharaux C, Dupont C, et al. Association between fibrinogen level and severity of postpartum haemorrhage: secondary analysis of a prospective trial. $\mathrm{Br} \mathrm{J}$ Anaesth. 2012;108(6):984-9. doi: 10.1093/bja/aes096.

8. de Lloyd L, Bovington R, Kaye A, et al. Standard haemostatic tests following major obstetric haemorrhage. Int J Obstet Anesth. 2011;20(2):135-41. doi: 10.1016/j.ijoa.2010.12.002.

9. Gayat E, Resche-Rigon M, Morel O, et al. Predictive factors of advanced interventional procedures in a multicentre severe postpartum haemorrhage study. Intensive Care Med. 2011;37(11):1816-25. doi: 10.1007/s00134-011-2315-0.

10. Solomon C, Collis RE, Collins PW. Haemostatic monitoring during postpartum haemorrhage and implications for management. Br J Anaesth. 2012;109(6):851-63. doi: 10.1093/bja/aes361.

11. Mallaiah S, Barclay P, Harrod I, Chevannes C, Bhalla A. Introduction of an algorithm for ROTEM-guided fibrinogen concentrate administration in major obstetric haemorrhage. Anaesthesia. 2015;70(2):166-75. doi: 10.1111/anae.12859.

12. Dietrich W, Faraoni D, von Heymann C, et al. ESA guidelines on the management of severe perioperative bleeding: comments on behalf of the Subcommittee on Transfusion and Haemostasis of the European Association of Cardiothoracic Anaesthesiologists. Eur J Anaesthesiol. 2014;31(4):239-41. doi: 10.1097/eja.0000000000000033.

13. Carvalho M, Rodrigues $\mathrm{A}$, Gomes $\mathrm{M}$, et al. Interventional Algorithms for the Control of Coagulopathic Bleeding in Surgical, Trauma, and Postpartum Settings: Recommendations From the Share Network Group. Clin Appl Thromb Hemost. 2016;22(2):121-37. doi: 10.1177/1076029614559773.

14. Charbit B, Mandelbrot L, Samain E, et al. The decrease of fibrinogen is an early predictor of the severity of postpartum hemorrhage. J Thromb Haemost. 2007;5(2):266-73. doi: 10.1111/j.1538-7836.2007.02297.x.

15. Wikkelso AJ, Edwards HM, Afshari A, et al. Pre-emptive treatment with fibrinogen concentrate for postpartum haemorrhage: randomized controlled trial. Br J Anaesth. 2015;114(4):623-33. doi: 10.1093/bja/aeu444.

16. Godier A, Susen S, Samama CM. Treatment of massive bleeding with prothrombin complex concentrate: argument against. J Thromb Haemost. 2010;8(12):2592-5. doi: 10.1111/j.1538-7836.2010.04062.x.

17. Martinez-Calle N, Marcos-Jubilar M, Alfonso A, et al. [Safety and efficacy of a prothrombin complex concentrate in patients with coagulopathy and hemorrhage]. An Sist Sanit Navar. 2014;37(3):363-9.

18. Ducloy-Bouthors AS, Mignon A, Huissoud C, Grouin JM, Mercier FJ. Fibrinogen concentrate as a treatment for postpartum haemorrhage-induced coagulopathy: A study protocol for a randomised multicentre controlled trial. The fibrinogen in haemorrhage of DELivery (FIDEL) trial. Anaesth Crit Care Pain Med. 2016;35(4):293-8. doi: 10.1016/j.accpm.2015.10.011.

Copyright (C) 2017 The Author (s); This is an open-access article distributed under the terms of the Creative Commons Attribution License (http://creativecommons.org/licenses/by/4.0), which permits unrestricted use, distribution, and reproduction in any medium, provided the original work is properly cited. 